\title{
HOMOMORPHS OF KNOT GROUPS ${ }^{1}$
}

\author{
DENNIS JOHNSON
}

\begin{abstract}
ABSTRACr. A simple proof that every group finitely generated by a single conjugacy class is the quotient of a knot group.
\end{abstract}

We are concerned with the following problem:

Given a group $G$, does there exist a knot group $\pi$ and a homomorphism of $\pi$ onto $G$ ?

This problem is known as Neuwirth's problem (see [N, Chapter 6, §4]). Since $\pi$ is finitely generated by the conjugates of a single element, namely of a meridian of the knot, $G$ must also be so generated if the problem is to have a solution; the question is then really if a $G$, finitely generated (f.g.) by one conjugacy class, ${ }^{2}$ is the homomorph of a knot group. Gonzalez-Acuña gave an affirmative answer to this question in [GA]; his methods are difficult however, involving 4-manifolds and nonsimply connected surgery. We give here a very simple and elementary proof of the result. ${ }^{3}$

First, we note that the requirements can be made slightly more stringent, as follows: $G$ is finitely generated by the conjugates of an element $\mu$, so we may ask for a solution knot group $\pi$ with meridian $u \in \pi$ and an onto homomorphism $h: \pi \rightarrow G$ which carries $u$ onto $\mu$. Our statement of the Theorem is then:

THEOREM. Let $G$ be a group finitely generated by the conjugates of $\mu \in G$. Then there is a knot with group $\pi$ and meridian $u \in \pi$, and a homomorphism $h$ of $\pi$ onto $G$ carrying $u$ onto $\mu$. $^{4}$

Before proving the theorem, we need some facts about homomorphisms of knot groups into $G$. We assume the knot to be given by a planar diagram of the usual sort, so its group $\pi$ is generated by the standard meridians (one for each overcrossing) of the type:

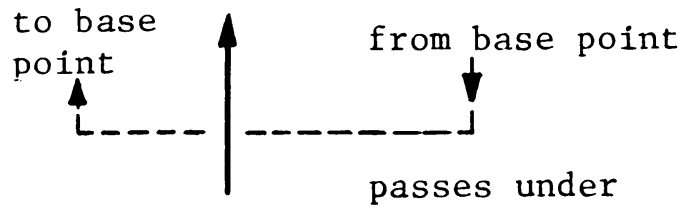

Received by the editors April 13, 1978 and, in revised form, July 25, 1978.

AMS (MOS) subject classifications (1970). Primary 55A25.

${ }^{1}$ This paper presents the results of one phase of research carried out at the Jet Propulsion Laboratory, California Institute of Technology, under Contract NAS7-100, sponsored by the National Aeronautics and Space Administration.

${ }^{2}$ This is equivalent to the simpler condition that $G$ is $f . g$. and generated by one conjugacy class.

${ }^{3}$ The referee has informed me that this proof was already known for some time to Andrew Casson.

${ }^{4}$ Gonzalez-Acuña also proved this result. 0002-9939/80/0000-0031/\$02.00 
This meridian can be designated by some letter attached to this overcrossing, and a homomorphism of $\pi$ into $G$ can be represented just by indicating (along this overcrossing) the image $g_{i} \in G$ of said meridian. The standard knot relations $y=z x z^{-1}$ hold at the crossing

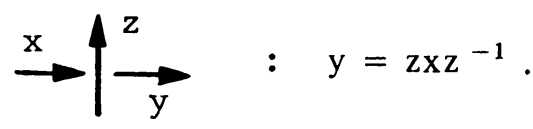

Thus a represented knot has elements of $G$ attached to its overcrossings which satisfy these relations in $G$ at every crossing.

We introduce now the notion of a represented band. By a band we mean, loosely, a pair of parallel, oppositely directed arcs of the diagram, with no other arcs of the knot passing between the two arcs of the band. That is, the band looks locally like one of the three types below (orientations may be reversed):
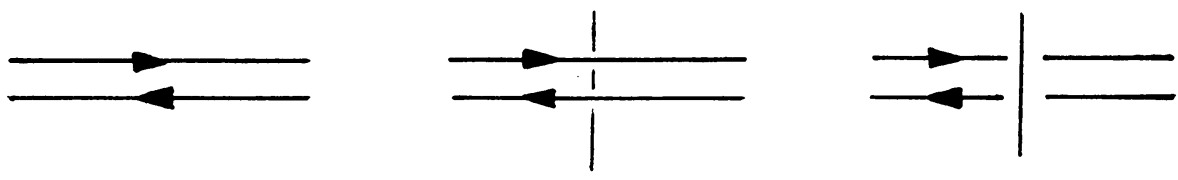

not:

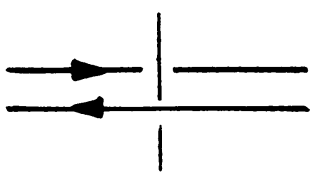

More precisely, we could define a band to be a rectangular strip $A B C D$, as in the figure below:

A

D

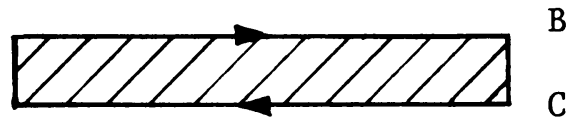

which has been imbedded in $S^{3}$ disjointly from the knot, excepting that the oriented arcs $A B, C D$ lie long the oriented knot. A represented band is a band with the same element of $G$ on both its arcs. Consider now what happens as a represented band crosses over or under some other arc of the diagram. Figure 1 shows the former, and we see that laying a band over an arc labelled $x$ does not change anything except between the arcs of the band. Figure 2 shows what happens when a band passes under a single arc: the equality of values on the two band-arcs is preserved, and thus a represented band remains a represented band in the process of passing under any arc. 


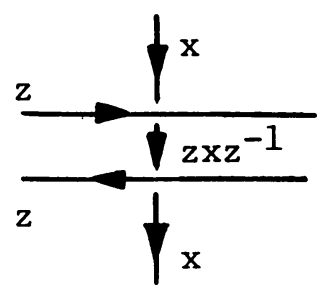

FIGURE 1

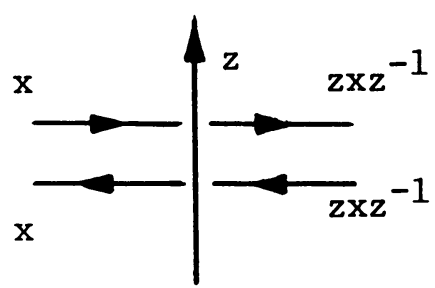

FIGURE 2

(Other cases, involving differently oriented arcs, are entirely analogous to the above.) These two figures indicate how we can alter a given represented knot or link diagram by drawing out a thin band from some arc of the diagram and weaving it through the previously established parts of the diagram, without essential change to the representation values there. We may then do band connections of the type:

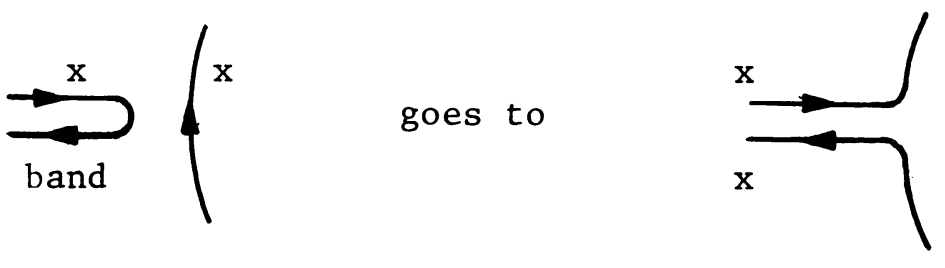

and still have a $G$ representation of the altered link. It is by this process that we construct the solution to the problem.

Proof of THE TheOReM. By hypothesis, $G$ is generated by the conjugates $\mu=\mu_{1}, \mu_{2}, \ldots, \mu_{n}$ of $\mu$. Since $\mu_{i}$ is conjugate to $\mu$, there exist words $W_{i}$ in the $\mu$ 's such that $\mu_{i}=W_{i} \mu W_{i}^{-1}$.

We start with a represented trivial link of $n$ components:
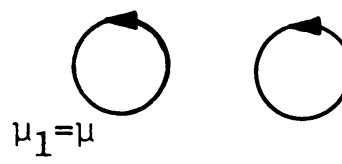

$\mu_{2}$

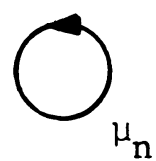

This representation is certainly onto $G$. Now $\mu_{2}=W_{2} \mu W_{2}^{-1}$. To illustrate our procedure, we will show how to proceed with the example $W_{2}=\mu_{3}$; the general case will then follow easily. For $\mu_{2}=\mu_{3} \mu \mu_{3}^{-1}$ then, we begin by drawing out a thin band from the first circle, and passing it through the 3rd as in the following figure.

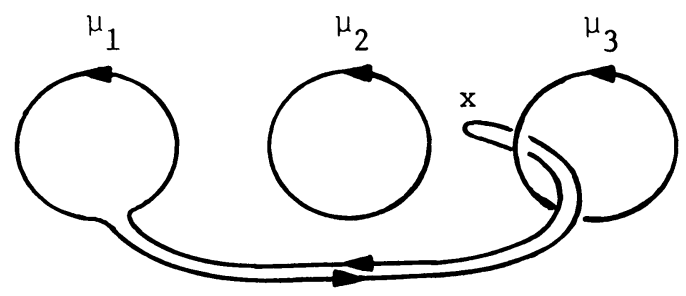


The value of $x$ on the new section of band is then $x=\mu_{3} \mu_{1} \mu_{3}^{-1}=\mu_{2}$. Hence we may connect this to the second circle as in the following figure:

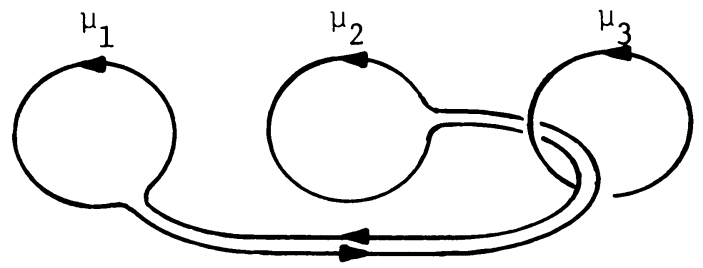

(it is easy to see that the orientations of the band and the circle always look like those in Figure 3, and so the band connection is permissible). Thus we have reduced the number of components by one, but the representation still involves all the generators, and so is still onto. If we had $W_{2}=\mu_{3}^{-1}$ instead of $\mu_{3}$, we would pass the band through the $3 \mathrm{rd}$ circle in the opposite fashion (under first, then over) to get $x=\mu_{3}^{-1} \mu_{1} \mu_{3}$; if $W_{2}$ is a word product of the various $\mu_{i}$ and their inverses, then we must pass our band through the corresponding various circles in the appropriate directions to get $x=$ $W_{2} \mu_{1} W_{2}^{-1}=\mu_{2}$. As the band advances it may be necessary for it to cross over or under previous parts of itself, but the laws illustrated in Figures 1 and 2 show that no essential alteration to these previous parts occurs. The same applies also if we now draw out a second band from the first circle, and weave it in such a way as to get $x=W_{3} \mu_{1} W_{3}^{-1}=\mu_{3}$, then connecting to the 3rd circle, etc. Thus eventually we have band connected all the components together, and we have a knot. The representation is onto $G$ and takes a chosen meridian (that of the first circle) to $\mu \in G$.

Note that the construction actually gives a ribbon knot; the original link bounds the obvious $n$ discs, and the bands intersect only these discs in the standard ribbon type intersections. Also, the Theorem as stated suggests immediately the following problem: suppose not only the meridian's image in $G$ be specified, but also the longitude's; does there exist a solution knot in this case? We will solve this problem in a forthcoming paper.

\section{REFERENCES}

[F] R. H. Fox, A quick trip through knot theory, Topology of 3-Manifolds and Related Topics (Proc. Univ. of Georgia Institute, 1961), Prentice-Hall, Englewood Cliffs, N. J., 1962, pp. 120-167.

[GA] F. Gonzalez-Acuña, Homomorphs of knot groups, Ann. of Math. (2) 102 (1975), 373-377.

[N] L. P. Neuwirth, Knot groups, Ann. of Math. Studies, no. 56, Princeton Univ. Press, Princeton, N. J., 1965.

Jet Propulsion laboratory, California Institute of Technology, 4800 Oak Grove Drive, Pasadena, California 91103 